\title{
Desarrollo del capital humano y la satisfacción laboral en la Universidad Privada de Huancayo Franklin Roosevelt, Huancayo - 2017
}

\author{
Development of human capital and job satisfaction in the Universidad Privada de Huancayo Franklin \\ Roosevelt, Huancayo - 2017 \\ Rosario Pilar Espinoza Landa ${ }^{1, a}$, Roy River Vilca Rodríguez ${ }^{1, b}$, Yumi Fiorella Bastidas Tocasca ${ }^{1}$, Kevin \\ Condor Trucios ${ }^{1}$
}

\section{RESUMEN}

La investigación "Desarrollo del capital humano y la satisfacción laboral en la Universidad Privada de Huancayo Franklin Roosevelt", buscó determinar como el desarrollo del capital humano contribuye a mejorar el nivel de satisfacción laboral de los colaboradores, identificando los niveles de desarrollo del capital humano y describir como estos se reflejan en la satisfacción laboral. La metodología empleada es de tipo básica teórica, con nivel descriptivo correlacional, se utilizó el método científico y con un diseño no experimental descriptivo correlacional simple causal. Las variables, capital humano y satisfacción laboral se correlacionan de manera significativa, evidenciándose un nivel significativo de desarrollo humano. El desarrollo del Capital humano contribuye significativamente a mejorar el nivel de satisfacción laboral de los colaboradores dándonos a entender que si no se contribuye con el desarrollo del capital humano no se lograrán los objetivos trazados debido a la insatisfacción del personal.

PALABRAS CLAVE: Desarrollo del capital humano, satisfacción laboral, remuneración, incentivo económico.

\section{SUMMARY}

The research "Development of human capital and job satisfaction at the Private University of Huancayo Franklin Roosevelt", sought to determine how the development of human capital contributes to improving the level of job satisfaction of employees, identifying levels of development of human capital and describe how these are reflected in job satisfaction. The methodology used is a theoretical basic type, with correlational descriptive level, the scientific method was used with a simple causal descriptive correlational descriptive design. With the variables, human capital and labor satisfaction, which are related in a meaningful way, evidencing a significant level of human development. It was concluded that the development of human capital contributes significantly to improving the level job satisfaction of employees, giving us to understand that if we do not contribute with the development of human capital, we will not achieve the objectives set due to staff dissatisfaction.

KEYWORDS: Human capital development, job satisfaction, remuneration, economic incentive. 


\section{INTRODUCCIÓN}

La Universidad Privada de Huancayo Franklin Roosevelt, cuya principal actividad es brindar servicios educativos universitarios de calidad, muy a pesar de sus esfuerzos se han identificado algunos problemas que impiden su desarrollo en el mercado de Huancayo una de ellas es la falta de valoración del capital humano ya que no se evalúa a los colaboradores de acuerdo a su desempeño laboral, más por el contrario parece haberse detenido las evaluaciones y ascensos de personal administrativo hecho que se ve reflejado en la insatisfacción laboral de los colaboradores.

Debido a ello la investigación tuvo como objetivo determinar cómo el desarrollo del capital humano contribuye a mejorar el nivel de satisfacción laboral de los colaboradores de la Universidad Privada de Huancayo Franklin Roosevelt Huancayo 2017. En el desarrollo de esta investigación se analizó el desarrollo Capital Humano que se define como: "el aumento en la capacidad de la producción del trabajo alcanzada con mejoras en las capacidades de trabajadores, estas capacidades realzadas se adquieren con el entrenamiento, la educación y la experiencia. Se refiere al conocimiento práctico, las habilidades adquiridas y las capacidades aprendidas de un individuo que lo hacen potencialmente" (Gary 2014).

Así como también la Satisfacción Laboral de los colaboradores de la Universidad, entendiéndose como "el conjunto de actitudes generales del individuo hacia su trabajo. Quien está muy satisfecho con su puesto tiene actitudes positivas hacia éste; quien está insatisfecho, muestra en cambio, actitudes negativas. (Robbins, 2009).

\section{MATERIALES Y MÉTODOS}

El análisis del desarrollo del capital humano y la satisfacción laboral en el ámbito empresarial es un tema de creciente relevancia. Entre las evidencias que justifican un análisis de este tipo están la dimensión de la actividad económica, la ausencia de competencias personales en la gestión de los servicios educativos, los cuales se ven justificados con la poca valoración del capital humano y la insatisfacción económica de los colaboradores de una institución universitaria, que como toda empresa privada busca el crecimiento económico mas no brinda mucha importancia al bienestar laboral y económico de sus servidores.
En ese sentido la Universidad Privada de Huancayo Franklin Roosevelt, cuya principal actividad es brindar servicios educativos universitarios de calidad a un sector joven de la población que viene en busca de un título profesional, es una institución donde se ha observado la problemática de la falta de valoración del capital humano ya que no se evalúa a los colaboradores de acuerdo a su desempeño laboral, más por el contrario parece haberse detenido las evaluaciones y ascensos de personal administrativo hecho que se ve reflejado en la insatisfacción laboral de los colaboradores, por lo que esta investigación buscará medir si la capacidad de la producción del trabajo es alcanzada con mejoras en las capacidades de los trabajadores, mejora sus actitudes en el contexto laboral que influirán de una manera significativa en los comportamientos y desde luego en los resultados.

Durante el desarrollo de la investigación se analizó el Capital Humano que implica: "Las capacidades productivas de las personas son las que importan porque con ellas se manipulan el capital económico, material e inmaterial de la empresa en función de su desarrollo y competitividad. Para lograr el óptimo desempeño del capital humano, se debe formar continuamente al personal, lo que genera bienestar y rendimiento laboral a través de la motivación y proporción de las herramientas adecuadas." (Aguilar, 2013).

Por otro lado, se analizó la Satisfacción Laboral definido como "El conjunto de sentimientos o actitudes favorables o desfavorables con que los trabajadores perciben sus puestos de trabajo", dicha actitud está basada en las creencias y valores que el trabajador desarrolla en su propio trabajo (Davis \& Newstrom, 1987)

\section{Problemas de la Investigación}

El investigador se planteó los problemas: ¿De qué manera el desarrollo del capital humano contribuye a mejorar el nivel de satisfacción laboral de los colaboradores de la Universidad Privada de Huancayo Franklin Roosevelt Huancayo 2017?, también ¿Cuáles son los niveles de desarrollo del Capital humano que se lleva a cabo en la Universidad Privada de Huancayo Franklin Roosevelt Huancayo 2017? Y ¿Cómo se refleja la satisfacción laboral de los colaboradores de la Universidad Privada de Huancayo Franklin Roosevelt Huancayo 2017 ? 
A estos problemas planteados se fijaron los objetivos: Determinar como el desarrollo del capital humano contribuye a mejorar el nivel de satisfacción laboral de los colaboradores de la Universidad Privada de Huancayo Franklin Roosevelt Huancayo 2017, como objetivos específicos: Determinar los niveles de Capital humano que se desarrollan en la Universidad Privada de Huancayo Franklin Roosevelt Huancayo 2017 y Describir como se refleja la satisfacción laboral de los colaboradores de la Universidad Privada de Huancayo Franklin Roosevelt Huancayo 2017.

Las hipótesis planteadas como posible solución a los problemas plateados fueron; El desarrollo del Capital humano contribuye significativamente a mejorar el nivel de satisfacción laboral de los colaboradores de la Universidad Privada de Huancayo Franklin Roosevelt Huancayo 2017.

El nivel de desarrollo del Capital humano es significativo en la Universidad Privada de Huancayo Franklin Roosevelt Huancayo 2017 y Los incentivos económicos reflejarían significativamente la satisfacción laboral de los colaboradores de la Universidad Privada de Huancayo Franklin Roosevelt Huancayo 2017.

\section{MATERIAL Y MÉTODOS}

La investigación utilizó la metodología basado en desarrollar la teoría acerca de Capital Humano y extenderla hacia la Satisfacción Laboral para relacionarla y ampliar teorías al respecto, con un Nivel de Investigación descriptivo Correlacional ya que describió las características importantes de las variables y su incidencia en la unidad de análisis, así también permitió conocer la relación que existe entre estas variables pretendiendo determinar la situación de estas en la muestra de la investigación.

La investigación tuvo el diseño No experimental Descriptivo Correlacional Simple causal, si las variables capitales humano y satisfacción laboral, se relacionan de una manera significativa, es decir, permitió conocer el grado en que las dos variables cambian de modo concomitante.

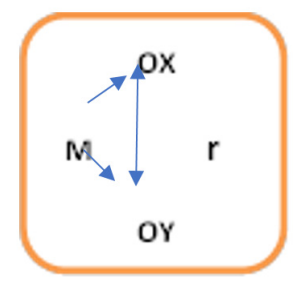

La Unidad de análisis de la investigación está determinada por el colaborador es decir trabajador administrativo a tiempo completo de la Universidad Privada de Huancayo Franklin Roosevelt. Por ende, la población de la investigación se conformó con 42 colaboradores administrativos de la Universidad Privada de Huancayo Franklin Roosevelt.

La técnica de muestreo utilizada fue el No Probabilístico por conveniencia debido a que la población de los colaboradores determinados como unidad de análisis son los mismos que formaran parte de la muestra, los cuales tienes las mismas características de ser trabajadores administrativos de la Universidad con contrato firmado y que prestan servicios a tiempo completo.

\section{RESULTADOS}

El desarrollo del Capital humano contribuye significativamente a mejorar el nivel de satisfacción laboral de los colaboradores de la Universidad Privada de Huancayo Franklin Roosevelt Huancayo 2017. Esta hipótesis se comprueba en la siguiente pregunta: ¿Recibe preparación necesaria para realizar el trabajo?, ¿La Universidad promueve el desarrollo del personal? Con estas preguntas el investigador pretendió identificar si la muestra recibe preparación que contribuya con su desarrollo personal y laboral, ya que una de las causas para que exista satisfacción en el capital humano es que la contantemente preparación para afrontar situaciones inesperadas, pudiendo así ser parte de la solución y brindar un mejor aporte a la institución, debido a ello en la encuesta aplicada a la muestra se tuvo como resultado:

Primera pregunta sobre si reciben preparación para realizar sus trabajos del $100 \%$ de los encuestados el $74 \%$ respondió que a veces recibe preparación necesaria para realizar el trabajo, el $14 \%$ manifestó que nunca recibe preparación necesaria para realizar el trabajo y el $12 \%$ dio como respuesta que siempre recibe preparación necesaria para realizar el trabajo. Segunda pregunta que contrasta la hipótesis es para saber si la Universidad promueve el desarrollo del personal, en esta pregunta se tuvo como resultado ante la hipótesis que del $100 \%$ de los encuestados el $67 \%$ respondió que a veces la Universidad promueve el desarrollo del personal, el $21 \%$ manifestó que siempre la Universidad promueve su desarrollo del personal y el $12 \%$ de los encuestados respondieron que nunca La Universidad promueve el desarrollo de la persona. Dando a entender que el capital humano está satisfecho, en un porcentaje poco considerable. 
Los incentivos económicos reflejarían significativamente la satisfacción laboral de los colaboradores de la Universidad Privada de Huancayo Franklin Roosevelt Huancayo 2017., en esta hipótesis se comprueban con las siguientes preguntas: ¿Considera que la remuneración económica es muy baja con relación a la labor que se realiza? y la segunda pregunta que también contribuye a la contrastación de la hipótesis es ¿No se siente a gusto con su jefe? Con estas preguntas el investigador explica si los colaboradores de la Universidad Roosevelt al recibir una remuneración o incentivo económico se sienten a gusto y satisfechos laborando en la universidad. Dado que el investigador considera, que un incentivo económico influye mucho en el desempeño laboral y debido a ello hay mejores resultados en el cumplimiento de tareas siendo estas productivas para la institución.

Del análisis de datos estadísticos se obtuvo los resultados; a la pregunta ¿Considera que la remuneración económica es muy baja con relación a la labor que se realiza? Del $100 \%$ de los encuestados el $57 \%$ considera que a veces la remuneración económica es muy baja con relación a la labor que se realiza, el 29\% considera que siempre la remuneración económica es muy baja con relación a la labor que se realiza y el 14\% considera que nunca la remuneración económica es muy baja con relación a la labor que se realiza.

A la segunda pregunta ¿No se siente a gusto con su jefe? Del 100\% de los encuestados el 53\% respondió que a veces no se siente a gusto con su jefe, el $43 \%$ manifestó que nunca se siente a gusto con su jefe y el $5 \%$ respondió que siempre no se siente a gusto con su jefe.

\section{DISCUSIÓN}

Para Gary (2014) el aumento en la capacidad de la producción del trabajo alcanzada con mejoras en las capacidades de trabajadores, estas capacidades realzadas se adquieren con el entrenamiento, la educación y la experiencia. Se refiere al conocimiento práctico, las habilidades adquiridas y las capacidades aprendidas de un individuo que lo hacen potencialmente.

Hoy en día las instituciones educativas están compitiendo en un mercado muy complejo, en donde los clientes muy exigentes ya no solo buscan educación certificada sino también nuevas experiencias. Entonces contar con personal capacitado y acorde con los cambios de las nuevas tendencias facilitará a que la universidad presente una imagen motivadora y atractiva para quienes ingresen a formar parte de ella, ya que, con preparar al personal para realizar sus trabajos dentro de la universidad, habrá un mejor desempeño laboral y a la vez transmitirá una imagen acompañada de una buena experiencia.

El personal feliz y satisfecho divulga lo que vivió en el trabajo haciendo ver que la Universidad promueve el desarrollo del personal. En tal situación se planteó la hipótesis en donde El desarrollo del Capital humano contribuye significativamente a mejorar el nivel de satisfacción laboral de los colaboradores de la Universidad Privada de Huancayo Franklin Roosevelt Huancayo 2017, la cual se contrastó con las repuestas a las siguientes preguntas (tabla 1 y tabla 2 ):

Tabla 1. ¿Recibe preparación necesaria para realizar el trabajo?

\begin{tabular}{lccc}
\hline Respuesta & Código & Frecuencia & Porcentaje (\%) \\
\hline Nunca & 1 & 6 & 14 \\
A veces & 2 & 31 & 74 \\
Siempre & 3 & 5 & 12 \\
TOTAL & & 42 & 100 \\
\hline
\end{tabular}

Tabla 2. ¿La Universidad promueve su desarrollo del personal?

\begin{tabular}{lccc}
\hline Respuesta & Codigo & Frecuencia & Porcentaje (\%) \\
\hline Nunca & 1 & 5 & 12 \\
A veces & 2 & 28 & 67 \\
Siempre & 3 & 9 & 21 \\
TOTAL & & 42 & 100 \\
\hline
\end{tabular}

Según Robbins (2009), el conjunto de actitudes generales del individuo hacia su trabajo. Quien está muy satisfecho con su puesto tiene actitudes positivas hacia éste; quien está insatisfecho, muestra en cambio, actitudes negativas. Cuando la gente habla de las actitudes de los trabajadores casi siempre se refiere a la satisfacción laboral; de hecho, es habitual utilizar una u otra expresión indistintamente.

Si bien es cierto un incentivo económico influye muchas veces en el desempeño laboral, en el cumplimiento de tareas y objetivos de la empresa. Siendo así que permite al personal estar satisfecho y motivado a seguir laborando. De ello la universidad obtendrá eficientes resultados, que se reflejen en el cumplimiento de los objetivos y metas trazadas. 
En tal situación se planteó la siguiente hipótesis, Los incentivos económicos reflejarían significativamente la satisfacción laboral de los colaboradores de la Universidad Privada de Huancayo Franklin Roosevelt Huancayo 2017 el cual se contrastó con las respuestas a las siguientes preguntas (tabla 3 y tabla 4 ):

Tabla 3. ¿Considera que la remuneración económica es muy baja con relación a la labor que se realiza?

\begin{tabular}{lccc}
\hline Respuesta & Codigo & Frecuencia & Porcentaje (\%) \\
\hline Nunca & 1 & 6 & 14 \\
A veces & 2 & 24 & 57 \\
Siempre & 3 & 12 & 29 \\
TOTAL & & 42 & 100 \\
\hline
\end{tabular}

Tabla 4. ¿No se siente a gusto con su jefe?

\begin{tabular}{lccc}
\hline Respuesta & Codigo & Frecuencia & Porcentaje (\%) \\
\hline Nunca & 1 & 17 & 43 \\
A veces & 2 & 21 & 53 \\
Siempre & 3 & 2 & 5 \\
TOTAL & & 40 & 100 \\
\hline
\end{tabular}

\section{CONCLUSIONES}

El desarrollo del Capital humano contribuye significativamente a mejorar el nivel de satisfacción laboral de los colaboradores de la Universidad Privada de Huancayo Franklin Roosevelt Huancayo 2017 nos da a entender que si no se contribuye con el desarrollo del capital humano no se lograrán las metas u objetivos trazados debido a la insatisfacción del personal, así mismo se debe tener presente que en las instituciones educativas están compitiendo en entornos donde el cliente es mucho más exigente y para satisfacerlos de forma eficiente veamos por el bienestar del capital humano.
El nivel de desarrollo del Capital humano es significativo en la Universidad Privada de Huancayo Franklin Roosevelt Huancayo 2017 se explica en que la universidad cuanto más desarrollo exista en el capital humano, mejores serán los resultados en cuanto al desempeño laboral y se lograrán los objetivos trazados siendo esta significativa para mejorar la situación actual de la Universidad Roosevelt.

Los incentivos económicos reflejarían significativamente la satisfacción laboral de los colaboradores de la Universidad Privada de Huancayo Franklin Roosevelt Huancayo 2017 se determina que un incentivo económico influye muchas veces en el desempeño laboral, en el cumplimiento de tareas y objetivos de la empresa con ellos el personal se encuentra más motivado a seguir laborando.

\section{Correspondencia:}

Dra. Rosario Pilar Espinoza Landa

Correo electrónico: respinoza@uroosevelt.edu.pe

\section{REFERENCIAS BIBLIOGRÁFICAS}

Aguilar, C. (2013). Capital humano. Ciudad de México: Region y Sociedad..

Davis, K. \& Newstrom, J. (1987). Comportamiento Humano en el Trabajo. Ciudad de Mexico: Editorial McGraw-Hill.

Gary, B. (2014). El capital humano y la producción. Madrid: Adventures-fox SA.

Robbins, S. (2009). Comportamiento Organizacional.

Ciudad de México: Editorial Prentice Hall Hispanoamericana.

Recibido: 15/11/2019

Aceptado: $\quad 02 / 03 / 2020$ 